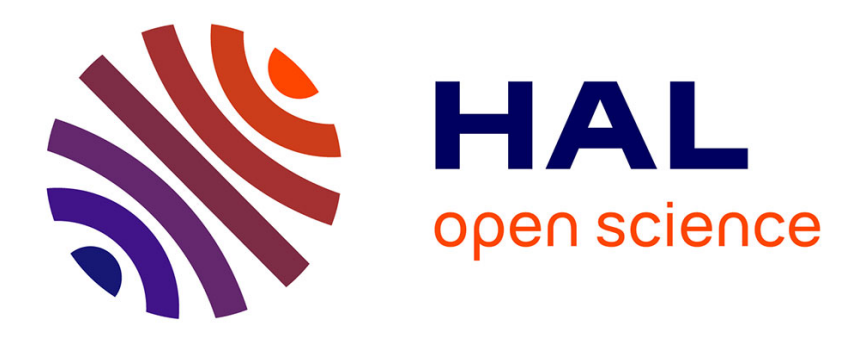

\title{
Une école européenne d'administration? L'improbable conversion de l'ENA à l'Europe
}

Michel Mangenot

\section{To cite this version:}

Michel Mangenot. Une école européenne d'administration? L'improbable conversion de l'ENA à l'Europe. Politix, 1998, 43, pp.7-32. halshs-00291207

\section{HAL Id: halshs-00291207 https://shs.hal.science/halshs-00291207}

Submitted on 27 Jun 2008

HAL is a multi-disciplinary open access archive for the deposit and dissemination of scientific research documents, whether they are published or not. The documents may come from teaching and research institutions in France or abroad, or from public or private research centers.
L'archive ouverte pluridisciplinaire HAL, est destinée au dépôt et à la diffusion de documents scientifiques de niveau recherche, publiés ou non, émanant des établissements d'enseignement et de recherche français ou étrangers, des laboratoires publics ou privés. 


\title{
Persée
}

http://www.persee.fr

\section{Une école européenne d'administration ? L'improbable conversion de l'ENA à l'Europe}

\author{
Michel Mangenot \\ Politix. Revue des sciences sociales du politique, Année 1998, Volume 11, Numéro 43 \\ p. 7 - 32
}

Voir l'article en ligne

«Une école européenne d'administration ? L'improbable conversion de l'ENA à l'Europe». Michel Mangenot [7-32], Loin d'être le fruit d'une stratégie délibérée de la part de sa direction, la conversion de l'ENA à l'Europe tient à l'origine aux investissements parallèles et non concertés de deux groupes d'énarques marginaux : des énarques au profil atypique et/ou dominé qui investissent l'Europe pour des raisons différentes et contribuent par là même à ouvrir un espace des carrières européennes ; les élèves étrangers, marginalisé au sein de l'Ecole, qui ont progressivement transformé le visage de l'ENA en revendiquant le titre d'«anciens". D'un côté comme de l'autre, ces acteurs ont alors contribué à façonner des représentations sur l'Europe, à nouer des liens et à construire des réseaux bruxellois dans lesquels peuvent aujourd'hui puiser les anciens élèves lancés dans des stratégies internationales désormais perçues comme légitimes et prestigieuses.

\section{Avertissement}

L'éditeur du site "PERSEE » - le Ministère de la jeunesse, de l'éducation nationale et de la recherche, Direction de l'enseignement supérieur, Sous-direction des bibliothèques et de la documentation - détient la propriété intellectuelle et les droits d'exploitation. A ce titre il est titulaire des droits d'auteur et du droit sui generis du producteur de bases de données sur ce site conformément à la loi nº $98-536$ du 1er juillet 1998 relative aux bases de données.

Les oeuvres reproduites sur le site «PERSEE » sont protégées par les dispositions générales du Code de la propriété intellectuelle.

Droits et devoirs des utilisateurs

Pour un usage strictement privé, la simple reproduction du contenu de ce site est libre.

Pour un usage scientifique ou pédagogique, à des fins de recherches, d'enseignement ou de communication excluant toute exploitation commerciale, la reproduction et la communication au public du contenu de ce site sont autorisées, sous réserve que celles-ci servent d'illustration, ne soient pas substantielles et ne soient pas expressément limitées (plans ou photographies). La mention Le Ministère de la jeunesse, de l'éducation nationale et de la recherche, Direction de l'enseignement supérieur, Sous-direction des bibliothèques et de la documentation sur chaque reproduction tirée du site est obligatoire ainsi que le nom de la revue et- lorsqu'ils sont indiqués - le nom de l'auteur et la référence du document reproduit.

Toute autre reproduction ou communication au public, intégrale ou substantielle du contenu de ce site, par quelque procédé que ce soit, de l'éditeur original de l'oeuvre, de l'auteur et de ses ayants droit.

La reproduction et l'exploitation des photographies et des plans, y compris à des fins commerciales, doivent être autorisés par l'éditeur du site, Le Ministère de la jeunesse, de l'éducation nationale et de la recherche, Direction de l'enseignement supérieur, Sous-direction des bibliothèques et de la documentation (voir http://www.sup.adc.education.fr/bib/). La source et les crédits devront toujours être mentionnés. 


\title{
Une école européenne d'administration? L'improbable conversion de l'ENA à l'Europe
}

\author{
Michel Mangenot \\ Groupe de sociologie politique européenne \\ Institut d'études politiques de Strasbourg
}

I

L EXISTE deux visions des rapports de l'ENA et de la construction européenne. La première, héroïque, est donnée par l'École ellemême depuis une dizaine d'années et consiste à présenter l'École comme aux avant-postes de l'Europe : «À Bruxelles, déjà, nous dit-on, "l'énarchie", par delà les clivages nationaux, fait l'Europe ou plutôt commence sans bruit à être l'Europe ${ }^{1}$, s'exclame Roger Fauroux, nouveau directeur, en $1987^{2}$. Plus largement partagée au sein de la haute fonction publique, la seconde vision souligne le manque d'intérêt de l'ENA (et du reste de l'ensemble des grandes écoles) pour l'Europe : «Les énarques, en dehors des cabineis des commissaires, soni pratiquement absents de la Commission. Des "Sciences po", des docteurs en droit abondent à tous les échelons de la catégorie $\mathrm{A}$, mais les anciens élèves des grandes écoles y sont rares", écrivait ainsi le correspondant du Monde à Bruxelles en $1993^{3}$.

Ces points de vue apparemment inconciliables n'ont pourtant rien d'exclusifs l'un de l'autre. Ils doivent tous deux leur part de vérité aux conditions très particulières dans lesquelles s'est opérée la "conversion européenne» de l'ENA. Rien n'était en effet moins évident que cette conversion. Confrontée, comme l'ensemble des autres grandes écoles, au

1. ENA Mensuel, $\mathrm{n}^{\circ}$ spécial, décembre 1987.

2. On peut se reporter plus largement sur ce point à l'analyse de H. Kaeble pour qui c'est dans la construction européenne que linfluence internationale du modele d'élite incarné par l'ENA doit être surtout recherchée («À propos des divers modeles d'élites», in Theret (B.), dir., L'Etat, la finance et le social. Souveraineté nationale et construction européenne, Paris, La Découverte, 1995, p. 129-130).

3. La Guérivière (J. de), Voyage a l'intérieur de l'Eurocratie, Paris, Le Monde Éditions, 1991, p.46. Voir aussi Kessler (M.-C.), «Les grands corps face à l'Europe», in D'Arcy (F.), Rouban (L.), dir., De la Ve République a l'Europe. Hommage d Jean-Louis Quermonne, Paris, Presses de Sciences Po, 1996, ou les grands corps sont eux aussi présentés comme ayant été longtemps hostiles ou passifs. C. Lequesne reconnaît également que les *analystes ont genéralement tendance à considérer que la haute fonction publique française a fait preuve, jusqu'au milieu des années 1980, d'une attitude méfiante à l'égard de la construction européenne», ( «L'administration centrale de la France et le systeme politique europeen : mutations et adaptations depuis l'Acte uniquen, in Meny (Y.), Muller (P.), Quermonne (J.-L.), dir., Politiques publiques en Europe, Paris, L'Harmattan, 1995, p. 144). 
nouvel environnement international et européen ${ }^{1}$, la position de l'ENA a longtemps pu apparaître comme antinomique avec les enjeux de la construction européenne. École nationale et surtout d'administration, elle est un des lieux privilégié de (re)production des représentations officielles (ou d'État) sur l'État ${ }^{2}$. Dans ces conditions, l'Europe n'a longtemps pas représenté un enjeu pour ses dirigeants, pas plus que pour la majorité des élèves. Il reste qu'une conversion à l'Europe s'est tout de même opérée, et ce comme malgré l'École.

Si les dirigeants actuels de l'ENA peuvent légitimement se prévaloir d'un fort ancrage européen, ils le doivent ainsi à des investissements qui relèvent de logiques autonomes, ou à tout le moins largement étrangères à l'École. Loin d'être le fruit d'une stratégie délibérée d'adaptation, la conversion européenne de l'ENA tient en effet aux investissements parallèles et non concertés à l'origine de deux types d'acteurs : les «énarques européens» (c'est-à-dire ayant investi dès l'origine les institutions européennes) et les élèves étrangers (qui ont quant à eux investi l'École). Les premiers, au profil atypique, sont partis tenter al'aventurem, et ont ainsi contribué à ouvrir un espace des carrières européennes aujourd'hui comblé par l'intérêt assez profondément renouvelé des élèves pour l'Europe. Les seconds, marginalisés au sein de l'institution, ont petit à petit transformé le visage de l'École en revendiquant leur titre d'«anciens" à part entière dans le même temps qu'ils accédaient à des postes importants de la construction européenne. D'un côté comme de l'autre, ces acteurs ont ainsi contribué à façonner des représentations, à nouer des liens et à construire des réseaux bruxellois dans lesquels peuvent aujourd'hui puiser les anciens élèves lancés dans des stratégies internationales désormais perçues comme légitimes et plus encore prestigieuses.

La coexistence des deux thèses sur le rapport de l'ENA à l'Europe apparaît en ce sens moins contradictoire qu'il n'y pouvait paraître. Elle tient à ce que l'ENA s'est davantage laissée convertir à l'Europe qu'elle ne s'y est convertie, comme si la position forte de l'École reposait principalement ici sur sa capacité à enrôler les plus marginaux de ses anciens. Aussi, est-ce les spécificité de cette conversion que l'on voudrait analyser ici en revenant sur les différents pôles, moments et effets de ces investissements. Au-delà du cas de l'ENA, il s'agira de mieux comprendre les raisons de cette tradition réputée française de l'administration européenne et, plus largement, ses effets dans la formation des «milieux» européens ${ }^{3}$.

1. Voir Lazuech (G.), «Le processus d'internationalisation des grandes écoles françaises», Actes de la recherche en sciences sociales, 121-122, 1998.

2. Les titres des livres consacres a l'ENA sont a cet égard explicites. Voir par exemple, Freches (J.), L'ENA : voyage au centre de l'Etat, Paris, Conti Fayolle, 1981 ; ou plus recemment Gaillard (J.-M.), L'ENA : miroir de l'Etat, de 1945 a nos jours, Bruxelles, Complexes, 1995.

3. Cet article repose sur un travail plus large mené dans le cadre d'un thèse de doctorat en cours sur l'Europe et la formation des hauts fonctionnaires français depuis 1948. 


\section{De «l'utilité» des marges \\ Les investissements européens des énarques français}

En dehors d'une phase d'incertitude aux origines de la construction européenne ${ }^{1}$, l'ENA n'a guère manifesté d'intérêt pour l'Europe. Mais ce serait rester prisonnier d'une vision trop homogène de l'École que de croire au désintérêt de tous les énarques. Par la diversité des investissements de ses membres et surtout leurs évolutions, l'ENA représente en ce sens un bon outil d'analyse des transformations subies par l'administration en France notamment sous l'effet de l'Europe. C'est ainsi que l'investissement européen des énarques français est d'abord l'histoire marginale de quelques individus au profil atypique ou dominés : de 1958 à 1988 , ils sont environ une vingtaine dans les institutions européennes, dont à peu près les deux tiers à la Commission. Mais cet investissement existe et favorise alors une entrée plus massive des énarques lors de deux moments forts de la politisation de la Commission: d'abord lors de la présidence de François-Xavier Ortoli, ensuite avec l'arrivée de Jacques Delors et la relance de la politique européenne de l'Acte unique.

\section{Linvestissement par les marges}

L'investissement des anciens élèves dans l'Europe est exceptionnel avant 1958. Seuls quelques énarques s'investissent dans la Haute autorité de la CECA et ils font aiors figure d'aventuriers ${ }^{2}$. Cetite aventure est plutôt brève pour l'initiateur, Jean Poincaré. Elle est liée de plus à une opportunité familiale qui renseigne sur le peu de cas qu'il est spontanément fait de l'Europe. Sorti de l'ENA sous-préfet en 1949, J. Poincaré arrive ainsi dès 1952 à Luxembourg, appelé par son oncle Léon Daume, major de l'X, l'un des deux premiers membres de la Haute

1. On trouvera plusieurs indicateurs d'un interet initial pour l'Europe. C'est le cas par exemple des sujets produits à l'occasion des premiers concours comme celui de 1948 («Quel rôle, d'après vous, demeure réservé à l'Europe dans le développement de la civilisation mondialew) et celui de 1949 ("L'unification europénne : rêve ou realite ?w). Si l'on en croit par ailleurs un ancien éleve sorti en 1949, la scolarité était à cette époque imprégnée d'un esprit européen : "Le grand mérite de l'ENA a été de nous apprendre à pardonner, sans oublier. Le cher commissaire de la République avait donné l'ordre aux professeurs d'exorciser la guerre larvée entre l'Allemagne et la France. Ça, c'était une ouverture sur l'Europe, le début de l'Europe» (entretien avec M. Blanc, prefet honoraire). Enfin en 1950, c'est l'ENA qui organise une première experience de formation commune, en accueillant pour quinze jours des fonctionnaires anglais, belges, hollandais et luxembourgeois, en application des dispositions culturelles du traité de Bruxelles. Par ailleurs, le fondateur de l'École, M. Debré, participe activement dans les années 19481950 aux débats fédéralistes au sein du Comité français pour l'Europe unie dans le cadre de l'ONU et publie un Projet de pacte pour une Union d'États europeens, Paris, Nagel, 1950.

2. Cette "mentalité d'aventure" semble courante chez les «eurocrates" et se trouve en phase avec la position sociale des élites européennes. Cf. Georgakakis (D.), "Les réalités d'un mythe : figure de l'eurocrate et institutionnalisation de l'Europe politiquen, in Dubois (V.), Dulong (D.), dir., La question technocratique, Strasbourg, PUS, à paraftre en 1999. 
autorité dde la $\mathrm{CECA}^{1}$. Devenu très rapidement directeur des relations extérieures, il préfêre suivre en 1958 Paul Delouvier, alors directeur des Finances, nommé délégué général du gouvernement en Algérie avant de «pantoufler». Second énarque à investir la CECA, Gérard Olivier n'est quant à lui pas de ceux qui ont suivi la voie royale. C'est par le concours interne qu'il est devenu administrateur civil au ministère de l'Intérieur. Arrivé en 1954, il fait carrière à la CECA et devient directeur du service juridique juste avant sa retraite. Le récit de son arrivée, racontée sur le mode de "l'aération" et du "hasard", est toutefois est bon indice du caractère peu naturel du parcours qu'il emprunte au sein des institutions européennes :
*C'est un peu par hasard, par l'intermédiaire de J. Poincaré. J'étais sorti de l'ENA dans la préfectorale ; j'étais d'abord chef de cabinet du prefet de Nancy, ensuite secrétaire général de la préfecture du Jura. Après deux ans, J. Poincaré m'a écrit qu'il était au service juridique après avoir été au cabinet de son oncle L. Daume. "Je vais quitter le service juridique pour passer au service des relations extérieures. Peut-être que la place pourrait t'intéresser...". Il m'a aménagé un rendez-vous à Strasbourg avec Michel Gaudet, membre du Conseil d'État, qui était le directeur du service juridique de la Haute autorité. Après diverses péripéties, j'ai été recruté et je me suis fait détacher de l'administration française à la CECA. À l'époque, mon idée était d'aller m'aérer quelque temps dans une institution internationale nouvelle et de revenir ensuite dans la préfectorale où je me plaisais. Finalement, je n'y suis jamais revenun" ${ }^{2}$.

D'autres énarques occupant des positions peu prestigieuses comme Jacques Leclerc, administrateur civil au ministère du Travail le suivent. Mais ce n'est qu'avec la création de la Commission, instituée par le Traité de Rome en 1958, que l'on assiste à l'arrivée de membres des grands corps : F.-X. Ortoli (1948), directeur général du Marché intérieur ; Alain Prate (1952), secrétaire du Comité monétaire ; JeanFrançois Deniau (1952), directeur de l'Association avec les pays tiers ; Jean Lévêque, directeur des finances à la Banque européenne d'investissement $^{3}$ (BEI) ; et Simon Nora (1947) qui devient directeur général de l'Économie de la CECA. En 1962, la direction du cabinet de la vice-présidence de la Commission, occupée par Robert Marjolin, revient par ailleurs à Robert Toulemon (1954), inspecteur des Finances, qui deviendra rapidement directeur général des Affaires industrielles. Enfin Michel Albert, inspecteur des Finances (1956), arrive en 1963 comme directeur-adjoint de la BEI puis devient directeur de la Structure et du Développement économique à la Commission.

1. J. Poincaré est par ailleurs le petit neveu de R. Poincare.

2. Entretien.

3. Sur la création de la Banque européenne d'investissement et le peu d'intérét qu'elle pouvait recueillir auprès du ministre des Finances de l'époque, voir les mémoires de Lévêque (J.), En premiere ligne, Paris, Albin Michel, 1986, p. 61, ou l'on apprend notamment son amitié avec F.X. Ortoli avec lequel il s'est entrainé pour le concours de sortie de l'ENA. 
Si la plupart d'entre eux sont aujourd'hui connus, ces premiers «énarques européens" issus de l'inspection des Finances sont à cette époque peu représentatifs de l'élite administrative française. Jeunes ce qui amuse d'ailleurs leurs collègues allemands qui les qualifient ironiquement de «jeunes génies français" ${ }^{1}-$, ils possèdent tous des caractéristiques rares ou marginales au sein de la haute fonction publique française ${ }^{2}$. Lorsqu'ils ne sont pas issus de milieu modeste (M. Albert) ou de l'entreprise (A. Prate, J. Lévêque), leur profil est assez atypique : F. X. Ortoli revient d'Indochine, R. Toulemon est un fédéraliste de la première heure. Quant à J.-F. Deniau il est mal à l'aise dans sa fonction, au point que l'Europe semble avoir tenu lieu d'échappatoire comme l'indiquent ses mémoires : «Jentre à l'Inspection et pars inspecter. Jai toujours préféré faire moi-même plutôt que de juger les autres. À défaut d'agir comme il me convient, j'écris comme il me plaît. Une série d'articles parus dans Combat sous le titre "Le règne des mots" me fait juger sévèrement par l'administration qui y voit une critique de l'ENA" ${ }^{3}$. Il poursuit de façon explicite sur son "choix" européen en indiquant combien l'Europe permet la valorisation de ressources et de croyances peu en vogue dans l'espace national : «Ma chance exceptionnelle est que la période 1955-1958 est celle de la grande relance de la construction de l'Europe. Le sujet, avec son mélange de technique et d'idéal, m'a toujours passionné. L'administration française n'a ni le temps ni le goût de tels exercices. Lâ place de nógociateur était largement à prendrem"

Cette présence d'énarques marginaux n'a au départ qu'un effet circonscrit sur les institutions européennes. Peu nombreux, les énarques se focalisent du reste sur quelques postes. Celui de directeur général du Marché intérieur, chargé de mettre en place l'union douanière, semble "réservé" aux hauts fonctionnaires français puisqu'il est occupé dans un premier temps par F.-X. Ortoli, puis par Pierre Millet (1947), administrateur civil, A. Prate et enfin R. Toulemon qui transforme d'ailleurs la direction générale ${ }^{5}$. Les postes dans les services juridiques semblent eux aussi convoités. Le cas de Jacques Mégret (1949), qui arrive en 1957 à la direction du service du Conseil est à cet égard exemplaire : membre du Conseil d'État, il doit en effet à l'ENA son ascension sociale, étant le fils d'un employé d'un modeste agent

1. Noël (E.), "Témoignage : l'administration de la Communauté europénne dans la rétrospective d'un ancien haut fonctionnairew, Annuaire d'histoire administrative européenne, 4, Baden-Baden, NomosVelarg, 1992, p. 152. Pour information, Deniau a 29 ans, Ortoli 32 ans.

2. De la même façon que les membres du groupe des «modernisateurs" de la haute fonction publique dans l'après-guerre possédaient des propriétés marginales. Cf. Gaiti (B.), "Histoire d'une renaissance. L'esprit de service public», Politix, 6, 1989, p. 64.

3. Mémoires de sept vies. Les temps aventureux, Paris, Plon, 1994, p. 419-420.

4. Memoires de sept vies. Croire et oser, Paris, Plon, 1996, p. 140.

5. Cette position est loin d'être négligeable dans la mesure ou l'union douanière est, avec la politique agricole commune, l'un des deux piliers de l'Europe communautaire des annees soixante. 
d'assurance $^{1}$. Mais pour le reste, ce sont des positions moins stratégiques qui sont investies à l'époque, comme par exemple la direction du Développement, ou le poste de directeur de la Sécurité sociale et des services sociaux qui est détenu par Jacques-Jean Ribas (1948) pendant plus de quinze années ${ }^{2}$. Dans le même temps, la représentation à Paris est elle aussi «occupée». Et là, l'indice de marginalité est encore plus net : Jacques Mallet qui devient en avril 1958 - avant même l'installation définitive de la Commission directeur-adjoint du bureau chargé du Marché commun a, en effet, démissionné dès sa sortie de l'ENA en $1952^{3}$.

Toutefois, et quand bien même leur présence exerce peu d'effet à Bruxelles, l'attrait de l'Europe en France apparaît transformé après le départ de cette première génération de "prétendants". Dès le milieu des années soixante, l'Europe devient ainsi une opportunité de carrière pour des agents aux positions homologues, comme les administrateurs civils qui rejoignent alors la Commission pour tenter leur chance. Ces derniers, on le sait, ne détiennent pas les positions les plus hautes dans la hiérarchie des corps. Ce sont donc encore des «seconds» qui investissent à cette époque les institutions européennes. Mais cet investissement n'en va pas moins contribuer à consolider la présence de l'administration française au sein des institutions européennes. Arrive ainsi Pierre Baichère, administrateur civil au ministère des Finances : affecté auprès de Claude Gruson au SEEF, il entre par la voie du cabinet de Maurice Lapie à la Haute autorité de la CECA dès 1960, devient en 1968 directeur des Budgets de la nouvelle Commission, puis en 1975 directeur général de l'Administration et du personnel. Arrive également Pierre Malvé, fils d'artisan sorti administrateur de l'ENA par le concours interne en $1960:$ il occupe pour commencer une position d'expert près la Commission avant d'intégrer l'institution en 1964, d'abord comme chef de bureau puis comme chef adjoint de cabinet. D'autres administrateurs du ministère des Affaires sociales ou détenant des postes sur le déclin, comme celui d'inspecteur de l'Économie nationale, font également leur entrée comme chefs de division, puis deviennent directeurs à la Commission. Ceux qui ont démissionné de la fonction publique française connaissent une carrière plus lente.

La nomination en 1967 de J.-F. Deniau au poste de commissaire européen ${ }^{4}$ ne tranche pas vraiment avec le caractère marginal de ces

1. Pour des éléments de biographie, on peut se reporter à une enquête sur son fils, $B$. Mégret, réalisée par Darmon (M.), Rosso (R.), L'après Le Pen, Paris, Seuil, 1998.

2. Sorti major de sa promotion, J.J. Ribas est issu d'une famille d'industriels.

3. N'ayant pas intégré un grand corps, il était jusque-là en charge des questions internationales au MRP.

4. A titre de comparaison, le premier enarque occupant un poste ministériel est, en 1958, V. Giscard d'Estaing comme secrétaire d'État aux Finances, lui aussi inspecteur des Finances. D'après N. Kauppi, le pourcentage d'anciens élèves de grandes écoles parmi les commissaires européens francais est important : 25\% pour l'ENA, $15 \%$ pour Polytechnique et $5 \%$ pour l'ENS («European Union Institutions in French Political [suite de la note page suivante] 
premiers investissements d'énarques. Car le profil de ce dernier est tout autant atypique que celui de ces prédécesseurs. Directeur général à la Commission, il démissionne en 1963, estimant qu'une page est tournée. À Paris, on ne lui propose aucun poste : il paye en retour son audace professionnelle et part en Mauritanie comme ambassadeur. Le ton qu'il emploie est à la mesure du peu de reconnaissance dont bénéficie à ce moment là les carrières européennes au sein de l'administration :

"Le directeur de cabinet du ministre des Finances explique à son ministre que me nommer a un poste important de l'administration nationale serait un défi vis-à-vis de ceux qui sont restés sagement à la maison au lieu de courir les océans lointains en enterrant des trésors sous les cocotiers. La bonne gestion du personnel, rue de Rivoli, est de considérer tout fonctionnaire français de Bruxelles comme ayant été rayé des cadres. C'est le contraire de la pratique de nos partenaires et bien sar des Anglais qui chercheront a placer dans l'administration européenne leurs meilleurs agents, avec promesse au bout de trois ans d'un avancement et du poste de leur choix. Si la France a perdu, après s'y être installée la première, le Canada, la Louisiane, les Indes, le Nigeria, le Brésil, la Floride, l'Australie occidentale et la NouvelleZélande, il doit bien exister une raison» ${ }^{1}$.

Il reste que, à partir des années soixante, le sens des investissements européens des énarques a changé, de même que les effets qu'ils produisent. D'une part, les énarques ont investi des positions (affaires économiques et monétaires, service juridique, direction du personnel) qui coniribueñit à ưưnnê à cette domière son style réputé frạnçais - et, ce d'autant plus que le normalien Émile Noël occupe désormais les fonctions de secrétaire général de la Commission. D'autre part, leur présence contribue à rendre plus visibles et surtout moins illégitimes les questions européennes au sein de l'administration française, au moment même où décline le dogme gaullien sur la construction européenne.

\section{La transition Ortoli}

Une étape particulièrement importante est franchie en 1973 lorsque F.$\mathrm{X}$. Ortoli accède à la présidence de la Commission. Cette nomination est un gage de reconnaissance importante des "énarques européens» et de leur rôle en Europe. Mais elle marque aussi une transformation de la structure de la haute fonction publique française à Bruxelles. Car si la nomination comme directeur général de cet inspecteur des Finances à peine âgé de 33 ans était un "pari», la figure d'Ortoli en 1973 tranche par rapport à ses prédécesseurs : symbole de la nouvelle génération de hauts fonctionnaires, F.-X. Ortoli a converti son audace professionnelle en capital politique en devenant, après son retour de Bruxelles en 1962, directeur de cabinet de Georges Pompidou, puis ministre en 1968. De ce

Careersw, Scandinavian Political Studies, 19 (1), 1996, p. 15). Le premier commissaire européen avec R. Marjolin, fut R. Lemaignen, saint-cyrien reconverti dans le commerce international et devenu l'un des dirigeants du CNPF.

1. Memoires de sept vies. Croire et oser, op. cit., p. 196. 
point de vue, sa nomination à la fonction de président de la Commission souligne l'importance gagnée par ce type de poste, comme en témoigne d'ailleurs l'investissement de nouveaux énarques qui la suit. Le cabinet de F.X. Ortoli est ainsi largement composé d'énarques; il en confie la direction à Philippe Jacquin de Margerie, maître des requêtes au Conseil d'État, issu d'une famille de diplomates, sorti en 1965. Par ailleurs, le profil des nouveaux entrants se «normalise», comme le confirme la nomination du camarade de promotion d'Ortoli, Claude Cheysson (fils d'inspecteur des Finances, ayant alors le titre d'ambassadeur), comme second commissaire français ${ }^{1}$. Déjà le passage de Jean-Claude Paye, diplomate, fils de l'ancien ministre Lucien Paye, comme chef de cabinet de Raymond Barre, vice-président de la Commission jusqu'en 1973, annonçait ce recentrage.

Des promotions restent encore possibles : c'est le cas de Raymond Phan Van Phi, administrateur civil, qui devient l'année même du retour d'Ortoli, directeur des Relations avec les organisations internationales, ou encore de P. Malvé qui devient directeur de l'Agriculture en 1975. Les domaines se diversifient dans le même temps que la présence des énarques se routinise au plus haut niveau des institutions européennes. C'est ainsi que le départ d'Ortoli de la présidence en 1977 n'empêche pas un jeune membre du Conseil d'État, Marc-André Feffer, sorti quatre ans plus tôt de l'ENA, et tout juste nommé maître des requêtes, de conserver la direction de ce cabinet. La politisation du recrutement n'est par ailleurs plus un phénomène rare, comme le montre, en 1977, la nomination à la Cour des Comptes européenne de Pierre Lelong, conseiller référendaire depuis 1965 mais surtout ancien député et ancien secrétaire d'État de 1974 à 1975.

\section{L'effet Delors}

Si à l'origine l'investissement européen est donc surtout l'affaire d'énarques qui refusent les voies traditionnelles de la carrière administrative, cet investissement s'inscrit très rapidement dans des stratégies professionnelles proprement carriéristes. À partir de 1985 et l'arrivée de J. Delors, l'Europe va, cette fois plus massivement, constituer un nouveau territoire de pouvoir pour les énarques.

C'est en effet J. Delors, autodidacte et ancien syndicaliste, qui va paradoxalement permettre une reconnaissance définitive de la place de l'ENA à Bruxelles. Professeur d'économie à l'ENA dans les années soixante ${ }^{2}$, le nouveau président de la Commission européenne a gardé pour cette institution une certaine vénération qui l'a poussé à

1. C. Cheysson, ambassadeur en Indonésie jusqu'en 1969, venait de passer trois ans en Afrique comme PDG de la Compagnie des potasses du Congo.

2. Appelé par P. Baucher, économiste de la planification et alors nouveau directeur des études de l'ENA, J. Delors est recruté en 1963 en même temps que M. Rocard. 
encourager vivement sa fille à suivre cette voie ${ }^{1}$. Il y "trouve» notamment Pascal Lamy ${ }^{2}$, jeune inspecteur des Finances de la même promotion que Martine Aubry (1975), également ancien d'HEC, qui sera son chef de cabinet à Bruxelles pendant dix ans. P. Lamy peut s'appuyer sur une nouvelle génération d'énarques qui accèdent alors progressivement à des postes clés. Le plus ancien est Jean-Louis Dewost (1964) : il a d'abord été, après seulement cinq ans au Conseil d'État, directeur adjoint de l'Organisation européenne pour la mise au point et la construction de lanceurs d'engins spatiaux, puis jurisconsulte au Conseil des ministres en 1979 ; il devient directeur général du Service juridique de la Commission en 1987. Le second, Jean-Paul Mingasson (1968), administrateur civil, directeur des Affaires monétaires en 1984, est nommé directeur général des Budgets en 1989. Il faut aussi ajouter Guy Legras (1967) qui devient en 1985 directeur général de l'Agriculture.

Tirant ainsi profit de l'espace des possibles ouvert par leurs aînés dans les années soixante, ces énarques européens incarnent un nouveau groupe. Ils sont intéressés à la réussite de la construction communautaire et tiennent désormais l'essentiel de leur notoriété de leur spécialisation européenne. Alors que leurs prédécesseurs concevaient l'Europe comme un passage, voire même pour certains un pis-aller, ces derniers n'entendent plus guère reprendre une carrière nationale. Parallèlement ils ont acquis des compétences qui se définissent à présent sur un mode strictement européen ei qui suritưưt sont reconnues comme telles, au point d'ailleurs d'être considérés l'un des nouveaux pôles de l'excellence européenne. Le départ à cet égard du normalien $\mathrm{E}$. Noël en 1987, après trente ans d'exercice, symbolise bien le relais pris par les énarques : de la même façon que l'École normale supérieure se situe sur le "pôle" descendant des grandes écoles en France, elle ne dispose plus au niveau européen de la place longtemps laissée vacante par l'ENA.

Avec la conjonction de cette nouvelle génération et du cabinet de la présidence, lieu d'attraction des ambitions européennes, l'investissement des énarques commence à s'amplifier. Stable jusque-là (autour de 20), le nombre d'énarques français dans les institutions européennes ne cesse d'augmenter : 35 en 1990, 40 en 1993 et 50

1. On peut se reporter sur ce point au chapitre "Naissance d'un crack» de la biographie parue sur M. Aubry, qui raconte que lors de son examen d'entrée à Sciences Po (section "Service public*) son père lui fit la recommandation suivante: «Ne dis pas que tu ne veux pas entrer a l'ENA, sinon ils vont te demander pourquoi tu as choisi l'option Service public* (Burel (P), Tatu (N.), Enquête sur une énigme politique, Paris, Calman-Levy, 1997 p. 44).

2. Maris (B.), «Revanche de la vie et des autodidactes, Jacques, admirateur de la haute fonction publique, hypertitrée, sera bientot prof à l'ENA (1963). Il y trouvera son Lamy, qu'il compare à Nora et Bloch-Lainé», in Jacques Delors, artiste et martyr, Paris, Albin Michel, 1993, p. 87. 
depuis $1995^{1}$. Le cabinet de J. Delors, surtout dans sa seconde phase (1990-1995), va faire en effet la part belle aux énarques ${ }^{2}$. En plus de P. Lamy et de Michel Hauswirth (1954), conseiller spécial, le cabinet compte ainsi quatre nouveaux énarques : Jean-Louis Vergnolle (1978), Jean-Pierre Jouyet (1980), Geneviève Pons-Deladrière (1982) et Fabrice Fries (1986).

Cette amplification se manifeste de plusieurs façons. De nouvelles positions sont tout d'abord conquises : il en va ainsi de la direction générale du Service juridique du Conseil occupé en 1990 par J.-C. Piris (1972), de la direction du Personnel de la Cour de justice, d'un poste à la direction générale de la Concurrence en 1994 obtenu par JeanFrançois Pons (1977), de la direction de la Politique extérieure et de sécurité commune avec Philippe Carre (1980), ou encore de la viceprésidence de la BEI avec Ariane Obolensky (1974). Autant de positions conquises auxquelles on peut également ajouter la présidence d'une autre institution européenne créée en avril 1991, la Banque européenne pour la reconstruction et le développement (BERD), à laquelle Jacques Attali accède ${ }^{3}$. La création du statut d'experts nationaux détachés permet par ailleurs à de nombreux énarques d'effectuer désormais leur mobilité à Bruxelles. Surtout, on assiste alors au début d'un phénomène nouveau : des énarques commencent à passer les concours des institutions européennes. Les cas se multiplient à partir de 1990 : l'exemple le plus révélateur est celui de Hubert Petit, sorti de l'École administrateur civil au ministère de la Santé en 1988, devenu administrateur à la Commission puis chef de cabinet à la Cour. Mais la question se pose alors de savoir s'ils peuvent garder leur qualité de fonctionnaire français, et accessoirement le titre d'énarque. Un dernier verrou saute alors : après un difficile débat le 3 juin 1994, suite à une demande du ministre de la Fonction publique qui l'interrogeait sur le sort à donner à un fonctionnaire détaché venant de réussir un concours, le Conseil d'État décide d'entériner la double appartenance ${ }^{4}$. Cette décision est précisée par une circulaire de la direction générale de l'Administration et de la Fonction publique du 9 novembre 1994 qui revient sur les raisons de ce «revirement parmi lesquelles est avancé «le souhait du gouvernement d'encourager les fonctionnaires à passer les concours communautaires pour renforcer la présence française à des niveaux de responsabilité conséquents tout en leur préservant une

1. On observe une évolution semblable du cóté des énarques députés européens : alors on n'en comptait qu'un seul en 1979, ils sont deux en 1984, huit en 1989 et treize en 1995.

2. Cf. la description que fait G. Ross du cabinet dans Jacques Delors and European Integration, Cambridge, Policy Press, 1995, p. 67-77.

3. Plus généralement, voir "Le rêve de gloire des fonctionnaires internationaux français", La Tribune de l'Expansion, 21 février 1991, dans lequel la journaliste s'exclame devant cette prédominance française dans les administrations multilatérales : «De quoi flatter l'orgueil national et l'amour-propre des directeurs de l'ENAn.

4. Sur ce débat juridique, voir Ruzié (D.), «La double appartenance à la fonction publique française et a la fonction publique internationalen, Journal de droit international, 122 (1), 1995. 
possibilité de retour dans l'administration, s'ils le souhaitent». Le choix d'une carrière européenne est ainsi facilité en rendant inutile la démission.

En 1975, Daniel Vignes disait ainsi à propos des fonctionnaires de nationalité française : «Moins marquée nous paraît être l'influence de l'ENA. Certes, Bruxelles compte nombre de brillants énarques, mais l'influence de leur école ne paraît pas directement dominante. Plus exactement, elle s'estompe devant le fait qu'en tout temps les Communautés ont pêché dans les différentes administrations nationales les spécialistes qu'il leur fallait. Ce sont des hommes rodés de certaines administrations, plus que des anciens élèves d'une école que l'on recrute ${ }^{1}$. Ce propos d'un non-énarque permet de mesurer l'étendue de la transformation qui s'est opérée en une quinzaine d'années seulement. À tel point que des propositions ont été faites lors du cinquantenaire de l'ENA, pour imaginer un recrutement direct à leur sortie de l'École ${ }^{2}$. De ce point de vue, la nomination la même année d'Yves-Thibaut de Silguy au poste de commissaire, à qui l'on confie l'un des dossiers les plus importants de la politique européenne - la mise en place de la monnaie unique - a valeur d'exemple. Ministre plénipotentiaire sorti de l'ENA en 1976, c'est un diplomate spécialiste des questions européennes qui est ainsi récompensé ${ }^{3}$. Cette nomination est l'aboutissement d'une présence des énarques affirmée depuis le milieu des années quatre-vingt, c'est-à-dire depuis la relance de la

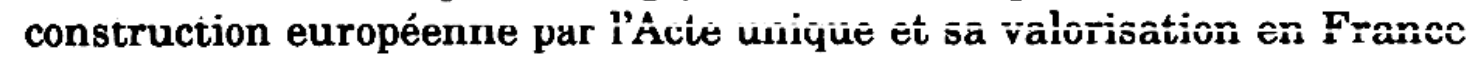
par la nouvelle volonté politique de François Mitterrand.

\section{L'ENA investie par les européens}

Parallèlement aux investissements des plus marginaux des énarques français, les élèves étrangers ont également joué un rôle important dans la conversion de L'ENA. Ce rôle des élèves étrangers apparaît même fondamental, au point par exemple qu'Odon Vallet, énarque devenu écrivain, reconnaissait en 1991 que «d'ores et déjà, [l'ENA] joue un rôle plus important pour la formation des fonctionnaires étrangers que pour celle des Français" ${ }^{4}$. Il reste que, là encore, le rôle des élèves étrangers apparaît comme le fruit de logiques autonomes. Car si ces derniers ont investi progressivement l'ENA, ils ont été marginalisés au sein de celleci. Aussi est-ce par un effet bien involontaire, dans l'optique de

1. Vignes (D.), «Les fonctionnaires communautaires de nationalite française», in Rideau (J.), Gerbet (P.), Torrelli (M.), Chevallier (R.-M.), dir., La France et les Communautés europeennes, Paris, LGDJ, 1975, p. 518.

2. Livre blanc du Cinquantenaire de l'ENA. Dix propositions pour l'administration de demain, Paris, Association des anciens eleves de l'ENA, 1995.

3 . On peut noter par ailleurs que le 25 septembre 1997 est organisée la première journée d'études de l'Association des anciens éleves de l'ENA à la Commission européenne, dont les principaux intervenants sont des énarques en poste a Bruxelles.

4. Le Point, 23 novembre 1991. 
contourner leur isolement, qu'ils ont créé les actuels réseaux européens de l'École.

\section{L'attraction d'une école}

"L'Europe nous envie les élites sorties de cette école», disait Yves Cuau dans une enquête sur l'ENA en $1967^{1}$. C'est là le paradoxe de cette institution : au moment où les critiques autour du complot des technocrates se font plus vives en France, les louanges affluent de toute l'Europe. De la même façon que les énarques ont, du moins dans un premier temps, peu investi la construction européenne alors que leur prestige était grand au sein de ces nouvelles institutions, l'École n'a pas profité de l'attrait qu'elle représentait pour les élèves étrangers.

Les premiers sont pourtant arrivés à l'École au titre d'auditeurs libres à la demande du ministère des Affaires étrangères et du ministère des États associés en 1949. Le concours d'entrée était alors réservé aux titulaires de la nationalité française mais certains auditeurs étaient acceptés, selon une procédure d'examen par le conseil d'administration. En 1953, le directeur de l'époque, Henri Burdeau de Fontenay, résume déjà bien toute la complexité de la position de l'École et les difficultés que pose cet accueil :

"Les activités de l'École sont donc assez diverses, les principales consistant à former des jeunes gens qui sont destinés à la fonction publique française et qui passent ses concours [...]. La gêne qui en résulte pour l'École ne doit pas être exagérée : il suffit, comme l'a d'ailleurs prescrit le conseil d'administration, de ne pas laisser augmenter dans de trop fortes proportions le nombre de ces eléments extérieurs, de façon que ne soit pas menacé l'équilibre interne de l'École. Quant aux réactions qui se manifestent parfois chez certains maîtres de conférences, notamment à la section des Affaires extérieures, concernant la présence à leurs enseignements de jeunes gens qui n'ont pas la qualité de citoyens français, elles s'atténuent toujours assez rapidement ; il semble d'ailleurs qu'elles ne se légitiment que dans une mesure insignifiante, les fonctionnaires chargés d'enseignement à l'École nationale d'administration n'ayant pas, à la vérité, l'occasion de communiquer à leurs auditeurs de véritables secrets d'État» ${ }^{2}$.

Il s'agit alors de contrôler ces eéléments extérieurs" qui proviennent encore essentiellement des pays de l'Union française. Petit à petit, cet accueil d'étrangers va s'élargir cependant aux Canadiens francophones puis aux Européens. La physionomie des auditeurs étrangers change ensuite considérablement à partir de 1966 en raison de la création de l'Institut international d'administration publique (IIAP), précisément chargé de la formation en France de hauts fonctionnaires étrangers. Voulu par le Général de Gaulle à la suite d'un long voyage en Amérique

1. Le Figaro, 3 aout 1967.

2. Rapport du directeur d son conseil d'administration, Paris, Imprimerie nationale, 1953. 
latine ${ }^{1}$, ce nouvel institut, conçu par le gouvernement comme une transformation de l'Institut des hautes études d'outre-mer, qui avait pris lui-même la suite de l'École de la France d'Outre-mer, précisait clairement, dans l'exposé des motifs de son décret fondateur, sa position vis-à-vis de l'ENA :

"La vocation profonde de l'École nationale d'administration ne peut être de concourir à la formation de fonctionnaires etrangers; ses programmes n'y sont pas adaptés. Destinée à des Français, l'ENA ne saurait, sous peine de se renier, se laisser déborder par un afflux d'étudiants étrangers. Elle peut donner, à titre tout à fait exceptionnel, un complément d'études de très haute valeur, comme le font d'ailleurs les autres grandes écoles françaises (Polytechnique, Normale supérieure, École spéciale militaire). Sans qu'il ne soit question de dénoncer les contrats déjà passés par certains pays étrangers avec l'École nationale d'administration, il est donc indispensable de créer un organisme nouveau, qui puisse coordonner, regrouper et concentrer les efforts accomplis en ce sens" ${ }^{2}$.

On perçoit mieux ici ce que doit la conversion de l'école à des investissements qui se sont joués contre elle et plus largement contre la volonté de l'État. Car c'est bien involontairement que, loin de respecter cet impératif gouvernemental, l'ENA se laisse au contraire déborder par un afflux d'élèves étrangers. Les jeunes fonctionnaires allemands vont en effet refuser d'être transférés à l'IIAP en arguant notamment du fait que le traité franco-allemand signé en 1963 par De Gaulle et Adenauer précisâit ưué c'était l'ENA qu ưi ćtait chargée d'accueillir les Allemands. Par ailleurs, François Gazier, directeur en 1965, relate une initiative du patronat allemand cette même année «venu trouver le directeur de l'École pour lui demander de venir exposer en Allemagne, devant un large public d'hommes politiques, de hauts fonctionnaires, d'industriels et d'étudiants, la formule sinon les secrets de cette École et de débattre avec eux de la possibilité d'introduire une école analogue en Allemagne fédérale" ${ }^{3}$. Finalement, l'ENA allemande ne se fera pas et le contingent d'élèves allemands va rapidement augmenter dès 1967.

L'effet de cette présence allemande est particulièrement important pour l'européanisation de l'ENA. Envoyés par leur autorités nationales, les auditeurs allemands sont en effet les représentants d'une stratégie précoce d'internationalisation de la fonction publique allemande. Nombre d'entre eux viennent ainsi à l'ENA pour devenir ensuite fonctionnaires européens, et c'est de cette façon que les «problématiques» européennes réintègrent l'École.

F. Gazier note à ce propos le urecrutement de très bonne qualité car c'étaient des Allemands qui avaient déjà des études juridiques assez

1. Voir le récit de F. de Baecque, alors directeur de cabinet de L. Joxe, dans «Comment est né l'IIAP ?n, Revue française d'administration publique, 40, 1986.

2. Décret du 2 décembre 1966.

3. Discours prononcé lors du colloque du Cinquantenaire de l'ENA, dont les actes ont été publiés dans La Revue administrative, 49, numéro spécial, 1996, p. 25. 
poussées et qui avaient le désir de devenir fonctionnaires européens pour la plupart. Pour devenir fonctionnaire européen, il était important qu'ils connaissent autre chose que la langue et le droit allemands. Venant à Paris, auprès de l'ENA, ils perfectionnaient leur français qui était en général bon d'ailleurs, ils avaient un bon niveau, c'est pourquoi l'enseignement était facile et rentable. Et ils avaient une connaissance des principes du droit français et de l'administration française qui ensuite leur permettait de faire carrière a Bruxelles dans de très bonnes conditions. Et beaucoup ont eu des postes importants ensuite dans les institutions européennes : ils étaient tout à fait qualifiés pour cela» ${ }^{1}$.

Le début de la contribution de l'ENA à une formation à l'Europe pourrait bien ainsi tenir d'un double «bricolage" : bricolage des fonctionnaires allemands qui complètent leur formation européenne par l'intermédiaire d'un enseignement qui ne leur est pas destiné ; bricolage de l'École, ensuite, qui s'efforce d'accompagner le mouvement. Car pour recevoir ces étudiants particuliers, l'ENA va alors inventer des formules diverses, mais sans moyens supplémentaires : cycles nationaux puis cycles spéciaux de présentation de l'administration française. Pour les élèves étrangers isolés au sein de l'École et ne partageant que très peu de cours avec leurs *collègues français», le moment le plus important demeure toutefois l'apprentissage sur le tas, et notamment le stage en préfecture $^{2}$.

Poussée comme malgré elle, l'École va enfin tirer profit de la concurrence avec l'IIAP récemment créé. L'existence de cette école et le refus des allemands de l'intégrer est de fait à l'origine d'un partage implicite des tâches entre les deux établissements, partage qui participe assez directement à la réorientation du rôle international de l'ENA vers l'Europe : à l'IIAP les pays en voie de développement, à l'ENA les pays européens, le Japon et l'Amérique du Nord ${ }^{3}$.

\footnotetext{
1. Entretien.

2. Méme si $P$. Racine, directeur des stages jusqu'en 1958 et devenu directeur de l'École en 1969 , explique en 1990 qu'il les a intégrés : «Je me suis beaucoup occupé des élèves étrangers allemands. On les avait mis a part des autres. S'ils viennent ici, c'est pour être avec des Français qui seront des gens importants plus tard. Je les ai intégrés avec leurs camarades français. Il faut le meme enseignement. Je veux qu'ils travaillent ensemble. L'objectif était de leur donner une formation commune et de développer l'esprit de compréhension pour plus tard. C'est énorme vous savez [...] C'était la volonté du général de Gaulle et du chancelier Adenauer : qu'ils soient ensemble. Les deux peuples principaux de l'Europe continentale devraient être formés en partie ensemblew (Archives orales du Comité pour l'histoire économique et financière, ministère de l'Économie et des Finances, 1990).

3. Un ancien éleve resume ainsi ce partage au sein du conseil d'administration de l'Association du 3 mai 1979 : «Dans le passé, l'École a accueilli pour des stages de duré très inégale des stagiaires de pays très divers. Mais depuis la creation de l'Institut d'administration publique, les stagiaires des pays en voie de développement sont accueillis par cet Institut et non plus par l'école. Celle-ci par contre a uniformisé, depuis la reforme de 1972, la durée des stages ouverts a des représentants des pays européens, nord-américains et du Japon» (compte-rendu du Conseil d'administration, Promotions, juin 1979).
} 
Lorsqu'encouragés par leur entrée dans la Communauté européenne, les Anglais affluent par la suite, ils trouvent ainsi une école qui non seulement possède déjà une marque européenne mais qui se trouve en outre plus prête à les accueillir. La voie tracée par les Allemands est ainsi réinterprétée dans un sens qui concerne plus directement l'Europe, l'École apparaissant désormais comme un modèle pour se former aux conséquences administratives de la Communauté : «Quand la GrandeBretagne a adhéré au Marché commun en 1973, le gouvernement anglais a demandé à la France, à Pompidou, d'envoyer à l'ENA soixante à quatre-vingt hauts fonctionnaires pour se familiariser avec la manière dont l'administration doit régler ses problèmes quand le pays fait partie de la Communauté», explique ainsi Pierre Racine, alors directeur ${ }^{1}$. Sans être encore labellisée comme école européenne, l'ENA est à partir de ce moment reconnue pour ses compétences en matières de politique européenne, même si on ne s'y forme pas à l'administration européenne mais à la gestion des relations avec Bruxelles².

\section{De la non reconnaissance au contournement}

Bien que la présence des étrangers est au principe d'un repositionnement partiel de l'école, cette présence ne s'accompagne pas d'une reconnaissance des étrangers. Cependant, cette distance maintenue à l'égard des étrangers va, là encore, représenter en quelque sorte une opportunité pour l'École. Pour pallier leur manque de reconnaissance, les anciens auditeurs ètrangers vont en eñet se lancer dans une entreprise de mobilisation collective qui va contribuer en retour à servir la présence européenne de l'École.

Si la direction de l'ENA a été hésitante dans l'accueil les élèves étrangers, l'Association des anciens élèves français est encore plus nettement réticente ${ }^{3}$. Se pose en effet pour elle le problème de la reconnaissance de la qualité d'anciens élèves aux auditeurs étrangers. Cette question, posée régulièrement par des stagiaires étrangers qui demande leur adhésion à l'Association des anciens élèves, est décisive pour une école qui, ne l'oublions pas, ne délivre aucun diplôme. Car le «trophée» qu'est l'étiquette d'ancien élève engage toutes les valeurs spécifiques de l'École. La sélection du concours sert d'ordination et consacre des méthodes de travail. Les épreuves de classement traduisent le sens hiérarchique. Bel exemple de ce que les valeurs deviennent des symboles, ce qui les maintient et les consolide, comme le dit Bailey ${ }^{4}$, la protection de ce titre exprime ainsi la défense de l'identité d'un groupe contre l'extérieur.

\section{Archives orales, op. cit.}

2. Ce qui ne sera pas sans conséquence par la suite dans la politique européenne britannique.

3. Sur ce point, cf. Moreau (C.), L'association des anciens de l'ENA, mémoire pour le DEA en Sciences administratives, Université de Paris Panthéon-Assas, 1994.

4. Bailey (F.-G.), Les règles du jeu politique. Etude anthropologique, Paris, PUF, 1971, p. 35. 
La réponse de l'Association est donc dans un premier temps négative et laisse apercevoir toute la distance des français à l'égard de leurs camarades : «Quelles que soient leurs qualités et l'estime que leurs camarades de promotion ont pour eux, il est indiscutable qu'ils ne répondent pas aux critères exigés pour être membres de l'Association : pas de concours de sortie, pas de classement dans un corps administratif et scolarité généralement réduite. Comment, dans ces conditions, les admettre à l'Association et refuser ceux qui, après une année de scolarité complète, n'ont pas subi avec succès le concours de sortie ? 1 .

À partir de 1979, une évolution est cependant perceptible au sein de l'Association qui renseigne assez bien sur les profits que l'École peut tirer des auditeurs étrangers. Pour le dire vite, si les stratégies internationales, et donc a fortiori européennes, n'étaient pas les plus payantes au sein de l'École, ceux qui les ont empruntées participent à une modification de leur perception qui invite l'Association à faire plus de cas des étudiants étrangers. Dans un éditorial de la revue Promotion, deux élèves français, Philippe Faure et Philippe Zeller, diplomates récemment sortis de l'École, le montrent très clairement lorsqu'ils expliquent pour la première fois les ubénéfices» qu'il est possible de tirer de la présence à l'École des étudiants étrangers :

«Venus de loin parfois, ils sont venus en nombre : plus de 600 , dont une majorité d'Allemands, de Britanniques, de Canadiens et de Grecs. D'abord stagiaires de cycles organisés spécialement à leur intention, ils ont été de plus en plus intégrés a la scolarité et aux activités des élèves français, et participent notamment aux travaux des séminaires. La présence à l'ENA de ces jeunes fonctionnaires étrangers est un exemple unique et précieux de coopération internationale dans le domaine, très réservé, de l'enseignement préparatoire à la haute fonction publique. Les bénéfices sont clairs : outre les liens d'amitié qu'ils tissent avec de futurs fonctionnaires français, nos hôtes font connaissance avec notre organisation administrative et nous apportent en échange leur propre expérience de la gestion publique. Il n'est pas abusif de dire qu'ils contribuent aussi au rayonnement de l'ENA à l'étranger. Quelques-uns d'entre eux, diplomates de formation, prolongent leur sejour à Paris par une affectation dans leur ambassade. Bref, le meilleur accueil doit leur être réservé» ${ }^{2}$.

Dès le début des années quatre-vingt des efforts sont ainsi consentis pour renforcer les liens avec les stagiaires étrangers. Des réceptions annuelles présidées par le secrétaire d'État aux Affaires étrangères ou par le ministre sont organisées. Mais le parrainage administratif, mis en place en même temps, n'aboutit pas faute de volontaires du côté français. Certains diplomates, peu écoutés, cherchent alors de nouvelles formules : «Il faudrait pouvoir créer, comme l'ont fait d'autres Associations d'anciens élèves, HEC par exemple, un réseau d'associations ou de groupes à l'étranger», propose ainsi P. Ziller en

1. Promotions, septembre 1974.

2. Promotions, décembre 1979. 
$1980^{1}$. Mais la discussion reste encore très vive sur le titre d'ancien élève toujours refusé ${ }^{2}$.

Rejetés de l'institution qui leur refuse leur titre, les anciens élèves étrangers vont dès lors s'attacher à la contourner en l'instrumentalisant : ils vont constituer leur propre regroupement. À la différence des autres grandes écoles qui ont créé comme HEC des groupes dans différents pays, suivant le modèle des universités américaines pour lesquelles le critère est de résider dans le pays, les anciens élèves étrangers de l'ENA ouvrent en effet - ils y sont en fait contraints - des groupes liés à leur nationalité. Les premiers seront les Allemands, qui lors d'un séminaire d'anciens stagiaires organisé par l'Office allemand des échanges universitaires à Bonn, en octobre 1983, se prononcent pour fonder une association des anciens stagiaires allemands. Une assemblée constitutive est prévue pour l'automne 1984. Au même moment, une Association des hauts fonctionnaires finlandais anciens stagiaires à l'ENA est lancée.

Ces initiatives ne sont pas sans effet, et ce d'autant moins qu'elles se produisent au moment même où de nouveaux énarques investissent l'Europe lors du mandat de J. Delors. D'abord, elles permettent une évolution du débat sur le titre d'anciens élèves. Le changement des intitulés est explicite : l'on passe de l'Association finlandaise des anciens élèves du cycle étranger à l'Association italienne des anciens de

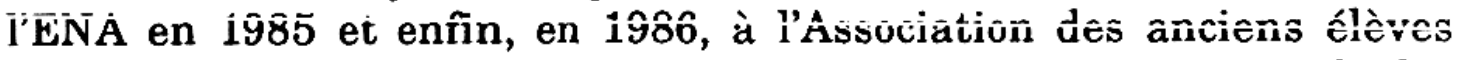
allemands de l'ENA. Ensuite, ces initiatives vont pouvoir tirer profit des positions importantes que les anciens élèves conquièrent dans leur pays. C'est tout particulièrement le cas des Allemands dont beaucoup entrent à cette période dans le cabinet du Chancelier Kohl. Ainsi Joachim Bitterlich de la promotion de 1976 entre à la cellule diplomatique du Chancelier en $1987^{3}$. Il est précédé de Johannes Ludewig (1974), chef du département économique et de Nobert Prill, de la même promotion, qui est chargé des discours et des déclarations. L'association allemande devient ainsi rapidement un groupe influent, comme en témoigne ce portrait d'un journaliste : «C'est une confrérie aussi influente que discrète. Elle compte 250 adhérents, dont 150 membres actifs. L'annuaire édité par l'association est un véritable Who's Who du pouvoir en Allemagne. Le président, Diethart von Preuschen, conseiller du groupe parlementaire FDP, est un ancien de la promotion Robespierre. C'est vrai, reconnaît-il, on a des gens partout.

1. Conseil d'administration du 27 octobre 1980, Promotions, décembre 1980.

2. Ils sont en effet inscrits dans l'annuaire à part. L'argument évoqué est celui d'une égalité de traitement avec les administrateurs des PTT et ceux de la ville de Paris, qui ont suivi une bonne partie de leur scolarité à l'ENA et qui ne sont pas recensés de la même façon.

3. Selon C. Lequesne, "cette arrivée facilite alors les relations franco-allemandes au sommet en particulier pendant la periode tendue de la réunification" (Une lecture décisionnelle de la politique européenne de François Mitterrand", in Cohen (S.), dir., La politique exterieure de François Mitterrand à l'epreuve de l'après-guerre froide, Paris, PUF, 1998, p. 136. 
Quand on décroche son téléphone pour appeler quelqu'un du réseau, on est sûr de recevoir un bon accueil» 1 .

Du coup des contacts se nouent avec l'Association française des anciens élèves. Une Confédération des associations françaises et étrangères est fondée le 21 janvier 1987 sous les auspices du ministre des Affaires étrangères et du ministre délégué chargé de la Fonction publique. Une réunion de la Confédération est alors prévue tous les ans, alternativement à Paris et dans un pays d'une Association étrangère. La réunion de Bonn en 1987, par exemple, voit le développement des contacts multilatéraux au sein de la Confédération qui devient «une véritable communauté internationale» 2 . Un grand colloque est enfin organisé par la Confédération en janvier 1992 : il porte sur «Les États modernes à la recherche d'une nouvelle efficacité». Pour Irène Bellier, «le plus surprenant de cette assemblée internationale était l'uniformisation du discours, du ton et du vêtement. Cela met en évidence l'existence d'un code formel de communication et de reconnaissance entre les cadres dirigeants au niveau international ${ }^{3}$.

Mais loin d'être un lieu consensuel à l'origine, cette confédération va en fait servir de lieu d'expression et de revendication des anciens élèves étrangers et particulièrement des européens, les plus actifs ${ }^{4}$, qui demandent un meilleur accueil des élèves à l'ENA. Si dans un premier temps l'École semble indifférente, ces revendications vont progressivement être entendues, en particulier par Roger Fauroux, devenu directeur de l'ENA en 1986. Celui-ci augmente de façon considérable les effectifs étrangers pour aboutir à un tiers de la promotion française. Et lorsqu'une nouvelle politique de formation continue est créée par René Lenoir en 1988 - le cycle international de formation permanente dans lequel les étrangers sont intégrés aux administrateurs civils nommés au tour extérieur ${ }^{5}$, ils revendiquent alors un droit de regard dans le recrutement des nouveaux élèves étrangers. Mais la principale requête reste une meilleure intégration dans les promotions françaises ainsi qu'une reconnaissance effective de leur passage à l'ENA. En 1990, ils obtiennent ainsi la création d'un diplôme spécifique : le diplôme international d'administration publique. Les dernières différences dans leur scolarité sont enfin levées en 1997.

\footnotetext{
1. Le Point, 15 juillet 1995.

2. Selon les propres termes du compte-rendu.

3. Bellier (I.), L'ENA comme si vous y étiez, Paris, Seuil, 1993, p. 320.

4. Sur ce point, voir Bellier (I.), «Le changement passe-t-il par l'École nationale d'administration ?", Politiques et management public, 8 (3), 1990, p. 60.

5. Ici encore, il faut noter que le développement du cycle international est le fruit d'une logique autonome. Car il s'inscrit dans une stratégie de la direction de la Formation permanente crée par la réforme de 1982 visant à légitimer son action, notamment par rapport aux deux autres directions qui se disputaient la charge des éleves étrangers, celle des Études et celle des Stages. Comme on assiste à partir de 1986 à une baisse des effectifs des promotions françaises, ces nouveaux cycles représentent un moyen de prendre position au sein des services de l'École tout en évitant une baisse globale des effectifs. Sur ces concurrences internes, Bellier (I.), L'ENA comme si vous y étiez, op. cit., 78-79.
} 
Ce qui n'empêche pas les élèves étrangers de conserver un regard critique vis-à-vis de l'ENA, comme en témoigne, par exemple, ces propos de Joachim Bitterlich tenus lors de la cérémonie de remise de diplômes en 1996 : «Il est vrai qu'aujourd'hui non seulement l'ENA, mais aussi le système français de formation des élites doivent réfléchir sérieusement à leurs orientations et leurs contenus» ${ }^{1}$.

\section{Une énarchie européenne?}

Les énarques dans la structuration des milieux bruxellois

«Une symbiose remarquable existe entre les élites françaises et la bureaucratie européenne, et cette symbiose ne résulte pas seulement des flux de personnels et de l'ouverture de carrières européennes nouvelles pour les fonctionnaires français, c'est-à-dire du simple élargissement à l'échelle européenne des pouvoirs de l'administration cette ouverture et cet élargissement valent, en effet, tout autant pour le personnel politico-administratif des autres pays -, mais aussi et surtout d'une forte identité en France et dans les Communautés européennes à Bruxelles des techniques de gestion et de l'esprit modernisateur et interventionniste», écrit Hartmut Kaeble ${ }^{2}$. Indépendamment du poids de l'École dans la fabrication d'un modèle français de l'administration, quelle est plus précisément sa part dans cette «symbiose» de la bureaucratie européenne et française ? Sans pouvoir prétendre apporter ici une réponse à cette question, on voudrait toutefois indiquer deux pistes de recherches en portant l'éclairage d'une part sur le travail de mobilisation menée par les associations d'énarques à Bruxelles et d'autre part sur les effets d'un tel travail.

\section{L'association européenne des anciens élèves}

Parallèlement aux mobilisations auxquelles ils se livrent dans leur pays d'origine, les anciens élèves étrangers vont contribuer à une affirmation de la présence de l'ENA au sein des institutions européennes. Avec les associations d'anciens dans neuf pays de la Communauté, des liens se sont en effet noués au sein des institutions européennes elles-mêmes. Un milieu spécifique s'est en quelque sorte constitué à Bruxelles. À la Commission, des réunions se sont par exemple organisées de manière informelle entre énarques français dès la fin des années soixante, sous l'influence de Pierre Baichère alors directeur général du Personnel. En 1973, au moment où Ortoli accède à la présidence, l'idée d'un rassemblement plus officiel apparaît plus nettement encore ${ }^{3}$. Mais c'est surtout avec l'action des nouvelles

1. «Une voie allemande», ENA Mensuel, février 1997.

2. Kaeble (H.) «A propos des divers modèles d'elites», art. cité.

3. Lors de l'Assemblée générale du 26 mars de l'Association des anciens élèves de l'ENA, un membre signale ainsi «que les anciens elèves en poste à Bruxelles au sein de la délégation française ont envisagé de créer une section particulière. C'est une bonne chose qui permettra de donner un caractère europén a notre Association et nous ne pouvons que nous en réjouir» (Promotions, mai 1973). 
associations d'anciens élèves étrangers que la structuration du milieu va se mettre en place.

L'initiative vient une fois de plus des Allemands - ce sont il est vrai les plus nombreux - qui organisent un grand rassemblement à Bruxelles en juillet $1990^{1}$. Tous les anciens élèves sont invités. Heike-Dagemar Joa, mise en disponibilité à la Commission depuis 1989 après avoir été élève de l'ENA en 1983-1985, intervient pour présenter l'ENA et l'esprit de ses anciens : «Bien que nos carrières soient différentes, bien évidemment, le fait d'avoir poursuivi la formation à l'ENA - être anciens - est un point commun. Nous attachons une grande valeur au fait que cet esprit surmonte les frontières. [...] La confédération internationale des associations des anciens élèves de l'ENA joue, à mon avis, un rôle important dans le cadre de la construction de l'Europe", avant de citer un extrait de la déclaration Schuman du 9 mai 1950 : "L'Europe ne se fera pas d'un coup, ni dans une construction d'ensemble : elle se fera par des réalisations concrètes créant d'abord une solidarité de fait».

C'est à partir de cette initiative qui permet de recenser les anciens élèves présents dans la capitale européenne que l'idée de concrétiser cette présence prend un nouvelle signification pour les énarques français. Dans une lettre du 21 novembre 1990, Louis Le Vert, membre de la représentation française à Bruxelles, demande ainsi à J. Delors d'accorder son haut patronage à une nouvelle association : l'Association européenne des anciens élèves de l'ENA. Une réunion constitutive est organisée au palais du Berlaymond le 24 janvier 1991 : elle est présidée par le président de la Commission. Selon ses statuts, elle a pour objet «de développer les liens entre les anciens élèves français et non français de l'ENA résidant en Belgique et au Grand-Duché de Luxembourg, de promouvoir par sa réflexion l'idée européenne et de contribuer au rayonnement de l'ENA, notamment par l'organisation de dîners débats, de colloques, de rencontres ou de toute autre manifestation culturelle de nature à réaliser son objet social» ${ }^{2}$. Il s'agit en fait d'une reconnaissance importante pour les élèves étrangers qui se voient reconnaître pour la première fois (même si implicitement) un titre équivalent à celui des Français.

1. Les Anglais se montrent également très actifs dans ce type de rassemblements. Parmi eux M. Donnely, dont le parcours est significatif : diplómé du Collège de Bruges avant d'entrer au Trésor en 1980, c'est son passage à l'ENA en 1983-1985 qui déclenche son investissement européen. A son retour à Londres, il devient le premier president de la nouvelle Association britannique des anciens élèves puis rejoint en 1989 le cabinet de sir L. Brittan, vice-president de la Commission.

2. Statuts de l'Association, Bruxelles, 30 janvier 1992. 
Constituée d'un ensemble de relations interpersonnelles ${ }^{1}$ et informelles ${ }^{2}$, on peut se demander si le groupe, aujourd'hui fort de cent cinquante personnes, n'est pas à présent disposé à fonctionner comme une communauté épistémique transnationale, c'est-à-dire un réseau de professionnels d'origines variées qui revendiquent une compétence particulière et construisent, sur la base de schèmes de perceptions communs, un espace de sens commun ${ }^{3}$. "Le petit cercle de l'ENA a un point commun de référence parmi ceux que l'on trouve dans la jeune Europe» reconnaissait ainsi un des membres. Et ce, alors même que ce groupe est loin d'être exclusivement composé de fonctionnaires : on y trouve des industriels, des banquiers, des avocats spécialisés et même un journaliste, correspondant à Bruxelles du journal Die Zeit. C'est que ce groupe se forge essentiellement autour de réunions-débats régulières, au cours desquelles l'information circule, les points de vue s'échangent et les visions du monde (et de l'Europe en particulier) convergent : «La plupart des discussions, réflexions que nous avons ensemble tournent autour des problèmes européens, plutôt communautaires. On fait venir les Commissaires les uns après les autres pour savoir ce qui se passe dans leur domaine. Et quand ce sont des hommes politiques, c'est autour des thèmes européens qu'ils viennent nous parler. C'est une occasion pour nous de constater qu'on participe un petit peu à la pensée unique en matière européennem ${ }^{4}$. Et de fait, le groupe a tendance à produire un discours unifié sur la construction européenne, qui se prolonge souvent à l'extérieur des enceintes communautaires. Le numéro spécial «L'Europe de 1992" d'ENA Mensuel paru en avril 1992 en est un bon exemple. En dehors de l'éditorial de J. Delors, tous les contributeurs sont membres de l'Association de Bruxelles ; surtout, ils comptent tous au nombre de ceux qui tiennent aujourd'hui le monopole de la production intellectuelle sur les questions européennes : JeanLouis Dewost y traite ainsi des problèmes juridiques, Guy Legras de la PAC, les Anglais des politiques économiques et Klaus Peter Schmid, le journaliste, de la question d'actualité, l'élargissement.

1. On assiste même au sein de ce groupe à des mariages entre énarques de différentes nationalités.

2. Le caractère informel du groupe l'oppose aussi à l'Association des français fonctionnaires des communautés européennes, crée en 1982, qui s'est engagée, après des revendications d'ordre statutaire, dans une politique de communication entre la fonction publique européenne et l'opinion publique française.

3. Haas (P.), "Introduction : Epistemic Communities and International Policy Coordination", International Organization, 46 (1), 1992 dans la méme revue Haas (P.), Adler (E.), "Conclusion : Epistemic Communities, World Older and the Creation of a Reflective Research Program*. Une présentation du concept est proposée par J. Richardson dans «Approches de la décision politique nationale et européenne fondées sur l'acteur : communautés de politique publique, réseaux par questions et communautés épistémiques", in Le Gales (Patrick), Thatcher (Mark), dir., Les réseaux de politiques publiques. Debat autour des policy networks, Paris, LHarmattan, 1995, p. 182-183.

4. Par ailleurs, la dimension européenne des différentes associations nationales d'anciens élèves est aussi affirmée. C'est ainsi par exemple que la nouvelle association des anciens élèves grecs est créé à l'occasion de la présidence grecque de l'Union europeenne en 1994. 
Enfin, avec l'autonomisation croissante de l'Association européenne qui développe son identité propre - une section s'est même ouverte en 1996 à Luxembourg -, un rapport d'extériorité est apparu vis-à-vis du siège parisien. J.-P. Mingasson, son président depuis le début, résume ainsi cette position : "C'est que dans tout ce dispositif, moi je suis aussi français que ne l'est le président de l'association parisienne et l'on nous considère comme si on était un petit peu en dehors" ${ }^{1}$. Le développement européen de l'ENA s'est ici effectué en dehors d'elle ou de ses représentants français.

\section{Une Europe marquée par l'ENA?}

Reste à mesurer ce que produit cette «association» d'énarques à Bruxelles, qu'ils s'agissent de ceux qui appartiennent à l'administration ou de ceux qui, lui étant extérieurs, interagissent avec elle. Plusieurs pistes peuvent être lancées à partir des discours produit aujourd'hui sur les énarques bruxellois, par eux-mêmes, les autorités de l'École ou encore les journalistes.

Le premier effet que semble avoir cette association est de légitimer l'Europe au sein de l'ENA. On l'observe notamment dans les publications de l'ENA, dans lesquelles les autobiographies d'anciens élèves étrangers se multiplient ; ou encore, plus généralement, dans un usage de leur titre d'ancien qui contribue à le faire entrer dans le cercle magique des titres légitimes au niveau européen. En bref, et du fait des investissements des étrangers, le titre d'ancien élève de l'ENA intègre la définition de l'excellence européenne. Les présentations de soi des anciens élèves dans ENA Mensuel en sont une bonne illustration.

À quelques mois d'intervalle, paraissent ainsi deux séries de portraits au titre explicite : «British ENArques in Europe ${ }^{2}$ tout d'abord, puis "Les Deutsche anciens en poste auprès de la CEE à Bruxelles se présentent" ${ }^{3}$. Tout se passe comme si le titre est ici clairement revendiqué pour accentuer une reconnaissance longtemps refusée, quand bien même les stratégies de présentation de soi varient selon la nationalité : si les énarques allemands se contentent de décrire strictement une carrière administrative dans ses aspects volontiers techniques, les Anglais livrent des souvenirs à partir desquels ils s'efforcent d'expliquer l'apport de l'École. C'est le cas du plus exemplaire d'entre eux, Martin Donnely, pour qui «la formation ENA est d'une utilité incomparable pour comprendre l'approche analytique de mes collègues européens. Il est important que des différences de style ou de présentation ne soient pas interprétées comme des dissentiments de fond" 4 . La mise en scène de son travail "qui lui donne des occasions de rencontrer des collègues et amis communs connus lors de son séjour à

1. Entretien.

2. ENA Mensuel international, novembre 1990.

3. ENA Mensuel international, mai 1991.

4. Ibid. 
l'ENA ; une récente délégation française au groupe de travail des services financiers du Conseil comprenait deux collègues de sa promotion", entend signifier la relation étroite entre l'École et le travail politique européen.

La présence même de ces portraits et autobiographies dans les colonnes de l'ENA Mensuel indique ensuite les profits que l'ENA retire de cette présence étrangère et renvoie en cela à une transformation plus générale du rapport de l'École à l'Europe. Car ces portraits affichés dans les publication de l'ENA constituent une vitrine des ressources possibles et nouvelles dans lesquelles les nouveaux énarques peuvent puiser. De ce point de vue, la façon dont est réécrite l'histoire des liens entre l'École et les étrangers est tout à fait instructive : l'action volontariste de l'Association française est désormais mise en avant, comme le montrent les propos ci-après de cet actuel responsable des activités internationales :

"La décision de l'association en 1984/1985, c'est de dire que du moment que des étrangers avaient suivi un an de scolarite en commun avec les Français, on pouvait considérer que c'était des anciens élèves de l'ENA. Il $y$ avait déjà dans l'annuaire les étrangers sous forme d'auditeurs étrangers : on a décidé que c'était des anciens élèves étrangers, dans le cadre de l'association, qui a tranché, et non l'École. Á l'époque, il y avait presque un millier d'étrangers qui étaient passés par l'ENA, dont on avait perdu la trace : on trouvait cela un peu absurde, a la fois pour des raisons professionnelles et conviviales. Et ça a été l'idée de Jacques Laureau, qui était vice-présidentît inteñinatioñal, de susciter cette constitution d'associations dans d'autres pays : on a commencé par l'Allemagne, parce que c'est là qu'il y avait le plus d'anciens. Et c'est bien une initiative de l'Association et non de l'École ou d'un pays"' ${ }^{1}$.

Cette réécriture de l'histoire peut s'analyser comme le produit de la "nécessité" pour l'École de sauver la face ou de faire «bonne figure" vis-à-vis de ses nouveaux et, surtout à présent, très utiles émissaires étrangers. En bref, l'existence d'une communauté d'énarques aux Communautés est devenu un atout à entretenir.

Troisième effet, la structuration de ce milieu d'énarques bruxellois fait que l'ENA intègre la définition d'un capital symbolique européen. Ce dernier peut notamment se lire dans la puissance qui est prêtée aux anciens énarques étrangers. Les commentaires journalistiques concernant l'existence d'une internationale énarchique peuvent être lus dans ce sens. Ainsi lorsque Sophie Coignard, auteur un an plus tôt un essai très critique sur les élites françaises ${ }^{2}$, écrit dans Le Point, à l'occasion de la création de la Confédération des associations d'anciens élèves, elle contribue à doter ce nouveau groupe des caractéristiques de la puissance : «Une véritable diplomatie parallèle regroupant huit cents ambassadeurs de quarante-huit nationalités différentes est ainsi

1. Entretien.

2. Coignard (S.), La Nomenklatura française, Paris, Belfond, 1986. 
consacrée. Après la mafia polytechnicienne et la fratrie des cabinets ministériels, l'internationale énarchique tisse son réseau hors des frontières» ${ }^{1}$. Pas étonnant dès lors que, malgré la teneur volontairement critique de l'article, les énarques français l'ait aussitôt reproduit dans ENA Mensuel ${ }^{2}$.

Si elle repose sur des intentions moins "critiques", la description de l'association européenne des énarques par Jean de La Guerivière, correspondant du Monde à Bruxelles, produit des effets semblables. Les formules telles que «nouvelle race d'européens» et «internationalisme entre gens qui savent» que ce journaliste emploie dans son article sont de ce point de vue assez éloquentes :

"Un après-midi de janvier 1991, au onzième étage du Berlaymont, une trentaine d'hommes et de femmes de toutes nationalités pénètrent avec plus ou moins d'assurance dans une des salles de réunion de la Commission. Tous n'appartenaient pas à la maison, mais la plupart l'avaient fréquentée un jour. Ils répondaient à l'invitation d'un des leurs, désireux de constituer une "amicale Belgique-Luxembourg" des anciens élèves de l'École nationale d'administration. Des Français, bien sâr, mais aussi des Britanniques, des Allemands, des Italiens. Douze associations regroupent les 1200 éleves originaires de toutes les parties du monde qui ont suivi le cycle étranger de l'École, et $M$. Raphaël Alormar, président de l'association française, se doutait bien qu'il devait y avoir une densité exceptionnelle d'énarques dans les parages, entre les institutions europeennes de Bruxelles et de Luxembourg, les "RP", les ambassades [...]. Étonnant spectacle que celui de ces retrouvailles internationales. Même les Britanniques parlent "énarque" avec les intonations, les tics et les élégances de langage de leurs camarades français. Ces grosses têtes passées dans les mêmes moules, ayant connu les mêmes expériences, donnent vraiment l'impression que - au niveau des élites - une nouvelle race d'européens est en train de naître, débarrassée des préjugés et des inhibitions de naguère, affranchie du discours nationaliste au profit d'un internationalisme entre gens "qui savent ${ }{ }^{3}$.

Montrés comme un groupe influent, les énarques autant français qu'étrangers peuvent en retour tirer profit de cette «qualification" pour rivaliser avec d'autres groupes au sein des milieux européens. Et ce d'autant plus que cette qualification prend sens dans un univers déjà marqué par la figure de l'Eurocrate, elle-même composante des représentations de la puissance et des ressources symboliques des professionnels de l'Europe ${ }^{4}$.

1. «Internationale énarchique», Le Point, 19-25 janvier 1987.

2. On peut encore citer des portraits plus récents d'énarques allemands qui vont dans le méme sens : «L'énarque qui conseille Helmut Kohl», Capital, mars 1996, et «Johannes Ludewig, un énarque en Allemagnew, Le Point, 4 mai 1996, où l'intéressé déclare entre autres : «Je ne suis pas une exception! Quarante ou cinquante de mes collegues du gouvernement fédéral ont fait l'ENAs.

3. La Guérivière (J. de), Voyage a l'intérieur de l'Eurocratie, op. cit., p. 44-45.

4. Cf. Georgakakis (D.), "Les réalités d'un mythe: figure de l'eurocrate et institutionnalisation de l'Europen, art. cité. 
En 1977, J.-F. Deniau choisit d'intituler son essai sur la construction européenne L'Europe interdite ${ }^{1}$. Dix sept ans plus tard, il en publie une nouvelle édition très légèrement remaniée sous le titre : La découverte de $l^{\prime} E u r o p e^{2}$. C'est dire si, de terre lointaine et délaissée par les énarques, l'Europe a changé de statut, au point que l'auteur peut à présent légitimement se présenter comme l'un de ceux ayant participé à sa conquête. Au delà, ce changement de titre indique bien la transformation du rapport de l'École à l'Europe.

Pour autant, cette transformation se comprend à l'analyse comme une conversion involontaire, du moins à l'origine. Car ce sont à chaque fois des acteurs «à l'extérieur» - les énarques devenus fonctionnaires européens - ou "extérieurs" - J. Delors, les élèves étrangers - qui ont impulsé l'ouverture de l'École. Dans cette conversion, qui doit bien sûr prendre à d'autres éléments, «l'externem a joué en quelque sorte contre "l'interne». Aujourd'hui l'ENA peut ainsi revendiquer avec facilité un nouveau positionnement européen. En l'absence d'une véritable institution européenne d'administration, elle peut même revendiquer le rôle imparti à l'origine au Collègue de Bruges : celui d'une école européenne d'administration ${ }^{3}$. C'est ainsi qu'en 1991, P. Lamy, dans une note de la Fondation Saint-Simon sur l'opportunité de la création d'une école européenne d'administration, répondait par la négative en mettant en avant le rôle international de l'ENA : «Je ne crois pas que ce soit une bonne idée. D'abord les différentes fonctions publiques ne sont

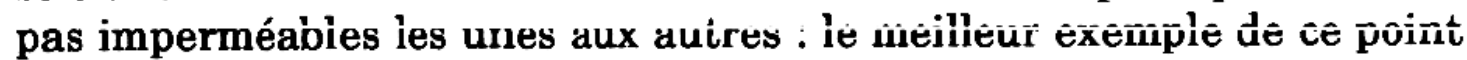
de vue est le réseau des anciens élèves étrangers de l'ENA en Allemagne ; il s'agit probablement d'un des meilleurs investissements dans le franco-allemand que nous ayons consenti..." ${ }^{4}$.

En 1992, la référence est explicitement affichée par l'ENA par la voix de son directeur, R. Lenoir : «L'ENA est ainsi devenue au fil des ans, une école européenne d'administration, à la fois utile aux Européens qui la fréquentent et aux ressortissants des autres continents qui viennent se familiariser avec la Communauté européenne ${ }^{5}$. Ce n'est pas le moment de la casser [...]. La délocalisation aboutirait à détruire un instrument de rayonnement de la culture administrative et juridique, de la langue française et de la formation en commun de la haute fonction publique européenne. Au moment même où il se bat pour la ratification des accords de Maastricht, comment le gouvernement pourrait-il mettre à

\footnotetext{
1. Paris, Seuil, 1977.
}

2. Paris, Seuil, 1994.

3. Présenté ainsi par'R. Dautry lors de son inauguration, le Collège a délaissé dans un premier temps cette fonction, avant d'ouvrir en 1974 une filiere administrative (cf. Formation et perfectionnement des fonctionnaires internationaux et européens, Bruges, Collège d'Europe, 1975, p. 6).

4. Entretien avec D. Olivennes intitule «Choses vues d'Europe», reproduit dans Esprit, $175,1991$.

5. C'est ici une manière de justifier l'ouverture de l'École aux élèves venus de pays lointains. 
mal un tel instrument ? ${ }^{1}$. C'est à l'occasion d'un moment de crise pour l'ENA que ce nouveau rôle est ainsi utilisé. Mais apparaissent alors les limites de cette conversion. La référence européenne n'est en effet pas seulement revendiquée sur le mode de la vocation; elle est aussi affichée pour refuser la délocalisation décidée en novembre 1991 par le gouvernement d'Edith Cresson, dans le même temps que cette décision est présentée comme un moyen d'accentuer l'européanisation de l'ENA.

\section{Annexe}

L'Europe à l'ENA

Chronologie des enseignements labelisés européens

Cours magistraux

1951 : «Les donnés politiques, économiques, sociales et démographiques de l'Unité europeennen (Guy de Carmoy et M. Doublet).

1953 : “Les institutions europénnes" : leur rôle politique, économique et militaire» (Guy de Carmoy).

1954 : «La Communauté européenne du Charbon et de l'Aciem (Paul Reuter).

\section{Seminaires restreints}

1957 : «Les échanges extérieurs français dans le cadre du Marché commun» (JeanFrançois Deniau).

1972 : «Affaires internationales*

- *L'influence de l'élargissement de la CEE sur ses relations avec les pays tiers* (Patrick O’Cornesse).

- «La politique agricole commune* (François Heilbronner).

- «Les conséquences de l'elargissement sur le fonctionnement des Communautés» (Claude Martin).

- «LEurope politique» (Gabriel Robin).

- «La Communauté économique européenne et la conférence sur la sécurité et la cooperation en Europen (Alfred Sieffer-Gaillardin).

- "Les relations de la Communauté élargie avec les pays de l'AELE non candidats» (Jean-Pierre Souviron).

1974 : «L'elargissement de la CEE» (Jean-Pierre Puissochet).

\section{Options}

1975 : «Droit communautaire» (Henri Mayras).

1979 : «Droit communautaire européen» (Jean Boulouis).

1980 : «Finances publiques communautaires» (Daniel Bouton, Denis Schramek).

1982 : «Problèmes financiers de la CEE» (Daniel Bouton).

1983 : «Droit communautaire et droit interne» (Jean Boulouis).

Modules complets d'enseignement (avec cours de cadrage et conferences)

1990-1994 : «Questions communautaires et internationales» (Bruno Joubert, coordinateur).

1992 : "La mise en oeuvre du traité de Maastricht et la construction européenne» (séminaire de promotion).

Depuis 1995 : «Procédures et affaires communautaires» (Gérard Druesne, coordinateur).

1. «L'ENA, un instrument d'influence françaisew, La Croix, 14 juillet 1992, repris dans ENA Mensuel, 225, novembre 1992, sous le titre «Un instrument d'influence française en Europe et dans le monde». 